\title{
DECOMPOSIÇÃO DO NO SOBRE Cu SUPORTADO EM ZEÓLITAS
}

\author{
Andréa Marins de Oliveira \\ Departamento de Engenharia Química, Universidade Federal do Rio Grande do Sul, \\ Rua Luiz Englert, s/n, 90046-900 Porto Alegre - RS \\ Sibele Berenice Castellã Pergher* \\ Departamento de Química, Universidade Regional Integrada do Alto Uruguai e das Missões, Campus Erechim, \\ CP 743, 99700-000 Erechim - RS \\ Celso Camilo Moro e Ione Maluf Baibich \\ Instituto de Química, Universidade Federal do Rio Grande do Sul, CP 15003, 91501-970 Porto Alegre - RS
}

Recebido em 10/3/03; aceito em 26/9/03

\begin{abstract}
NITRIC OXIDE DECOMPOSITION ON COPPER SUPPORTED ON ZEOLITES. Direct decomposition of NO on copper supported on zeolite catalysts such as MCM-22 and Beta was compared with that on the thoroughly studied Cu-ZSM-5. The catalysts were prepared by ion-exchange in basic media. They were characterized by atomic absorption, surface area, nitrogen adsorption at $77 \mathrm{~K}$, X-ray diffraction and temperature programmed reduction. The products of the reaction were analyzed by Fourier transform infrared spectroscopy using a gas cell. Catalytic activity tests indicated that zeolite catalysts, like Beta and MCM-22, lead to NO conversion values comparable to ZSM-5.
\end{abstract}

Keywords: NO decomposition; zeolites; copper.

\section{INTRODUÇÃO}

A crescente preocupação com a qualidade do ar e com o meio ambiente levou à adoção de regulamentações mais rigorosas para controlar a emissão dos gases de combustão provenientes de automóveis e de motores estacionários, em diversos países no mundo. Regulamentos ainda mais estritos serão introduzidos num futuro próximo. Os óxidos de nitrogênio, $\mathrm{NO}_{\mathrm{X}}$, são as principais causas da poluição do ar devido à formação de fumaça fotoquímica e da chuva ácida $^{1,2}$.

O uso de catalisadores é uma das formas de diminuir a emissão destes gases devido à sua transformação em gases inertes ${ }^{1,3}$.

Os catalisadores usados atualmente para automóveis com motores à gasolina são os chamados catalisadores de três vias ("Three Way Catalysts", TWC) que usam Pt/Rh. Os mesmos são bastante efetivos para os limites de emissões gasosas permitidos atualmente, porém requerem que a relação ar/combustível seja mantida próxima do ponto estequiométrico ${ }^{1,4}$.

Nos últimos anos, vários sistemas catalíticos têm sido estudados para a reação de decomposição do NO, especialmente catalisadores baseados em metais preciosos $(\mathrm{Pt}, \mathrm{Au}, \ldots)$ e materiais zeolíticos modificados com metais de transição $(\mathrm{Cu}, \mathrm{Co}, \ldots)^{5-9}$.

Neste sentido, o presente trabalho tem como objetivo o estudo de diferentes suportes zeolíticos, ZSM-5, MCM-22 e Beta, na preparação de catalisadores de cobre, para a reação de decomposição dos óxidos de nitrogênio, $\mathrm{NO}_{\mathrm{X}}$, através da avaliação da conversão do NO.

\section{PARTE EXPERIMENTAL}

As zeólitas ZSM-5, MCM-22 e Beta utilizadas neste trabalho foram fornecidas pelo Instituto de Tecnologia Química de Valência

*e-mail: pergher@uri.com.br
(Espanha), e foram preparadas segundo os procedimentos descritos na literatura ${ }^{10-13}$.

\section{Preparação dos catalisadores}

A ativação das zeólitas precursoras foi realizada a $500{ }^{\circ} \mathrm{C}$ por $6 \mathrm{~h}$ em forno convencional, com taxa de aquecimento de $2,5^{\circ} \mathrm{C} / \mathrm{min}$. Antes de chegar na temperatura desejada, o material passou por dois patamares: o primeiro a $150{ }^{\circ} \mathrm{C}$ por 60 min e o segundo a $350{ }^{\circ} \mathrm{C}$ por $90 \mathrm{~min}$.

Os catalisadores foram preparados usando $5 \mathrm{~g}$ de cada zeólita, realizando troca iônica com $200 \mathrm{~mL}$ de uma solução de nitrato de cobre $12 \mathrm{mmol} / \mathrm{L}$ sob agitação magnética durante $24 \mathrm{~h}$, à temperatura ambiente. Após esta etapa, elevou-se o pH da mistura reacional até 7,5 com solução de hidróxido de amônio $3 \mathrm{~mol} / \mathrm{L}$, deixando sob agitação por mais $1 \mathrm{~h}$. Filtrou-se, e o material resultante foi seco sob vácuo à temperatura ambiente por $24 \mathrm{~h}$. Calcinou-se em mufla por 3 h a $500{ }^{\circ} \mathrm{C}$, usando taxa de aquecimento $10^{\circ} \mathrm{C} / \mathrm{min}$. Este procedimento é uma adaptação da metodologia descrita primeiramente por Iwamoto et al. $^{14}$. Os materiais foram denominados tal como mostra a Tabela 1.

Tabela 1. Denominação dos catalisadores usados no estudo

\begin{tabular}{lcc}
\hline Zeólita & $\mathrm{SiO}_{2} / \mathrm{Al}_{2} \mathrm{O}_{3}$ & Catalisador \\
\hline ZSM-5 & 53 & $\mathrm{CuZ50}$ \\
ZSM-5 & 75 & $\mathrm{CuZ80}$ \\
MCM-22 & 30 & $\mathrm{CuM} 30$ \\
MCM-22 & 100 & $\mathrm{CuM} 100$ \\
BETA & 25 & $\mathrm{CuB25}$ \\
\hline
\end{tabular}

\section{Caracterização dos catalisadores}

Os padrões de difração de raios X (DRX) foram obtidos usando um difratômetro Siemens D 500, radiação $\mathrm{Cu}(\mathrm{K} \alpha), \lambda=1,5403 \AA$, 
corrente de $35 \mathrm{~mA} \mathrm{e} 2^{\circ}<2 \theta<74^{\circ}$, em uma velocidade de varredura de $1,2 \circ 2 \theta / \mathrm{min}$.

As análises de absorção atômica foram realizadas usando um espectrômetro de chama, marca VARIAN modelo AA55, em amostras $\left(50 \mathrm{mg}\right.$ ) pré-calcinadas a $950{ }^{\circ} \mathrm{C}$ por $3 \mathrm{~h}$ e dissolvidas com $1 \mathrm{~mL}$ de $\mathrm{HF}(40 \%)$ à temperatura ambiente, em frascos de polipropileno hermeticamente fechados e posteriormente diluídas a $100 \mathrm{~mL}$.

A área superficial e o volume de poros foram calculados a partir das isotermas de adsorção de nitrogênio a $77 \mathrm{~K}$, usando um aparelho Quantachrome modelo Autosorb 1MP, de amostras degasadas a $300{ }^{\circ} \mathrm{C}$ por $8 \mathrm{~h}$.

As análises de redução à temperatura programada (RTP) foram realizadas, em equipamento construído no DEQ/UEM, nos catalisadores frescos através da elevação gradual da temperatura, em fluxo de $1,75 \%$ de $\mathrm{H}_{2}$ em $\mathrm{Ar}$ até $610^{\circ} \mathrm{C}$, com programação de temperatura de $10{ }^{\circ} \mathrm{C} / \mathrm{min}$.

\section{Atividade catalítica}

A avaliação da atividade catalítica foi realizada em pressão atmosférica usando a conversão de 500 ppm de NO/Ar em um reator de fluxo contínuo e constante. A detecção de reagentes e produtos foi feita num espectrômetro de infravermelho com transformada de Fourier acoplado a uma célula de gás. Os cálculos da conversão foram feitos segundo metodologia desenvolvida por Dallago ${ }^{15}$, empregando as regiões do infravermelho descritas na Tabela 2. Antes de cada experimento os catalisadores sofreram calcinação a $500{ }^{\circ} \mathrm{C}$, em ar sintético, por $3 \mathrm{~h}$ e, em seguida, redução a $300^{\circ} \mathrm{C}$ em fluxo de $5 \%$ de $\mathrm{H}_{2} /$ Ar. A massa de catalisador usada variou entre 100 e $150 \mathrm{mg}$ e o fluxo usado foi de $100 \mathrm{~mL} / \mathrm{min}$.

Tabela 2. Regiões do espectro no infravermelho usadas para a identificação de compostos no estudo de atividade catalítica

\begin{tabular}{cc}
\hline Compostos & Intervalos $\left(\mathrm{cm}^{-1}\right)$ \\
\hline $\mathrm{NO}$ & $1955-1790$ \\
$\mathrm{NO}_{2}$ & $1658-1565$ \\
$\mathrm{~N}_{2} \mathrm{O}$ & $2266-2159$ \\
\hline
\end{tabular}

\section{RESULTADOS E DISCUSSÃO}

\section{Caracterização dos catalisadores}

A Tabela 3 mostra os teores de $\mathrm{Cu}$ e $\mathrm{Al}$ obtidos e o nível de troca iônica, considerando que um íon de cobre divalente compensa a carga negativa de dois íons de alumínio ${ }^{16,17}$. Observa-se, na tabela, que houve diferentes níveis de troca, sendo que o mais baixo está em torno de $20 \%$ e, em alguns casos, teríamos uma troca acima de $100 \%$ considerando que o $\mathrm{Cu}$ está como $\mathrm{Cu}^{+2}$. Trocas superiores a $100 \%$ são indicativo de que poderíamos ter espécies $\mathrm{Cu}^{+1}$ ou $\mathrm{CuOH}^{+}$.

Tabela 3. Análise química dos catalisadores

\begin{tabular}{lcccc}
\hline Catalisador & $\begin{array}{c}\text { Teor de } \\
\mathrm{Cu}(\%)\end{array}$ & $\begin{array}{c}\text { Teor de } \\
\mathrm{Al}_{2} \mathrm{O}_{3}(\%)^{*}\end{array}$ & $\begin{array}{c}\mathrm{Cu} / \mathrm{Al} \\
(\text { molar })\end{array}$ & $\begin{array}{c}\text { Troca } \\
\text { Iônica }(\%)\end{array}$ \\
\hline CuZ50 & 3,2 & 3,1 & 0,83 & 166 \\
CuZ80 & 2,7 & 2,2 & 0,98 & 196 \\
CuM30 & 2,7 & 5,3 & 0,41 & 89 \\
CuM100 & 0,21 & 1,7 & 0,10 & 20 \\
CuB25 & 3,3 & 6,3 & 0,42 & 84 \\
\hline
\end{tabular}

* Obtida pela relação $\mathrm{SiO}_{2} / \mathrm{Al}_{2} \mathrm{O}_{3}$ supondo que o material só tenha $\mathrm{SiO}_{2}$ e $\mathrm{Al}_{2} \mathrm{O}_{3}$.
Na Figura 1 são apresentados os difratogramas de raios X para as amostras preparadas e originais. Todos os catalisadores, após preparação e calcinação em forno convencional a $500{ }^{\circ} \mathrm{C}$ por $3 \mathrm{~h}$, apresentaram padrões de difração de raios $\mathrm{X}$ concordantes com a topologia dos materiais precursores: ZSM- $5^{10}$, MCM-22 $2^{11,12}$ ou Beta ${ }^{13}$. Observa-se que a troca do íon sódio pelo cobre não afetou de forma perceptível a integridade da estrutura zeolítica. A perda da cristalinidade poderia ser evidenciada pela formação de uma elevação na linha de base, na região compreendida entre $2 \theta=10-40^{\circ}$, centralizada em $2 \theta=22^{\circ}$, ou pelo aumento no ruído da linha de base.
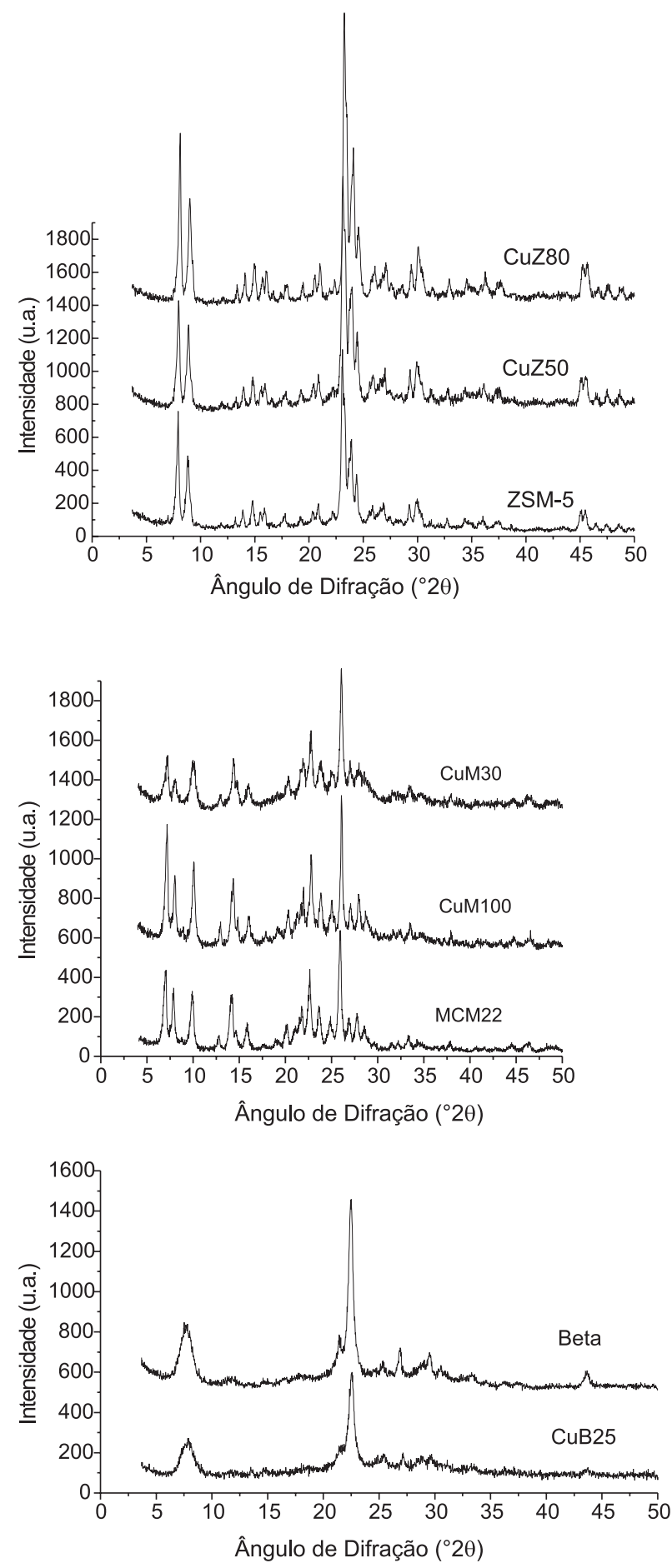

Figura 1. Difratogramas de raios $X$ dos materiais antes e após a troca iônica com cobre 
Não se observam sinais relevantes na região $2 \theta$ igual $36^{\circ}$ e $39^{\circ}$ referentes a $\mathrm{CuO}$ e $\mathrm{Cu}_{2} \mathrm{O}^{18}$, indicando que não houve formação de agregados de óxidos de cobre na superfície das zeólitas e nas cavidades das mesmas havendo, portanto, uma boa distribuição do íon cobre no interior da estrutura zeolítica.

A área superficial BET e o volume de poros dos catalisadores determinados a partir das isotermas de adsorção de nitrogênio à $77 \mathrm{~K}$ apresentaram os valores mostrados na Tabela 4 .

Tabela 4. Valores de área superficial específica e volume específico de poros

\begin{tabular}{lcccc}
\hline Catalisadores & $\begin{array}{c}\mathrm{A}_{\mathrm{BET}} \\
\left(\mathrm{m}^{2} / \mathrm{g}\right)\end{array}$ & $\begin{array}{c}\mathrm{A}_{\text {micro }} \\
\left(\mathrm{m}^{2} / \mathrm{g}\right)\end{array}$ & $\begin{array}{c}\mathrm{V}_{\text {TOTAL }} \\
\left(\mathrm{cm}^{3} / \mathrm{g}\right)\end{array}$ & $\begin{array}{c}\mathrm{V}_{\text {micro }} \\
\left(\mathrm{cm}^{3} / \mathrm{g}\right)\end{array}$ \\
\hline ZSM-5 (50) & 422 & 278 & 0,59 & 0,11 \\
CuZ50 & 330 & 216 & 0,50 & 0,09 \\
ZSM-5 (80) & 388 & 205 & 0,27 & 0,09 \\
CuZ80 & 383 & 234 & 0,26 & 0,10 \\
MCM-22 (30) & 453 & 312 & 0,53 & 0,16 \\
CuM30 & 444 & 333 & 0,50 & 0,14 \\
MCM-22 (100) & 480 & 386 & 0,37 & 0,16 \\
CuM100 & 445 & 364 & 0,34 & 0,15 \\
Beta (25) & 558 & 350 & 0,59 & 0,16 \\
CuB25 & 540 & 346 & 0,50 & 0,16 \\
\hline
\end{tabular}

Os resultados exibem boa concordância com os apresentados na literatura ${ }^{19}$; observa-se que a incorporação de cobre nos materiais provoca uma perda de área superficial BET e diminuição no volume total de poros. Estas perdas são ocasionadas principalmente pela diminuição da microporosidade nos materiais, evidenciada pela diminuição do volume de microporos. Este efeito é maior no catalisador preparado a partir da ZSM-5 (50) e sugere que, ao incorporar o cobre na estrutura microporosa, ocorre um ligeiro bloqueio de parte dos microporos, diminuindo sua acessibilidade. Já no catalisador preparado a partir da ZSM-5 (80), a área superficial praticamente não muda com a incorporação de cobre, mas ocorre um aumento da contribuição de microporos. Uma explicação para este fato poderia ser dada pois como este material já de partida contém mesoporosidade, acredita-se que o cobre estaria preferencialmente nesta mesoporosidade que é mais acessível durante a incorporação do mesmo, não bloqueando desta forma os canais microporosos. $\mathrm{O}$ aumento de área devido aos microporos sugere que a incorporação de cobre estaria, de certa forma, favorecendo a formação de microporos, possivelmente em posições de defeitos da rede cristalina.

Os perfis da redução à temperatura programada das amostras são apresentados na Figura 2. Da literatura obtemos que diversos experimentos de RTP de catalisadores de $\mathrm{Cu}$ suportado mostram que a redução do cobre pode ocorrer em dois estágios, apresentando dois picos de redução. Segundo alguns autores ${ }^{20-22}$ o primeiro pico corresponde à redução de $\mathrm{Cu}^{+2} \mathrm{a} \mathrm{Cu}^{+1} \mathrm{e}$ o segundo pico, de $\mathrm{Cu}^{+1} \mathrm{a} \mathrm{Cu}^{\circ}$. Entretanto, quando o cobre está bem disperso, como no caso de cobre suportado em zeólitas por troca iônica, a redução de $\mathrm{Cu}^{+}$a $\mathrm{Cu}$ metálico ocorre a temperaturas altas, geralmente acima de $327^{\circ} \mathrm{C}^{21-25}$. Através de experimento de RTP de catalisadores 3,1\% Cu/Na-ZSM-5, Sárkány et al. ${ }^{23}$, afirmam que quando se obtém dois máximos de redução a temperaturas inferiores a $337{ }^{\circ} \mathrm{C}$, o primeiro se deve à redução de espécies $(\mathrm{Cu}-$ $\mathrm{O}-\mathrm{Cu})^{+2}$, enquanto que o segundo está ligado à redução de espécies $\mathrm{Cu}(\mathrm{OH})^{+}$.

Comparando estes resultados com os obtidos em nosso trabalho, verificamos um pico a aproximadamente $277^{\circ} \mathrm{C}$ presente em todos os materiais, que é atribuído à redução da espécie $\mathrm{Cu}(\mathrm{OH})^{+}$. Antes deste pico observamos um ombro a aproximadamente $255^{\circ} \mathrm{C}$ que

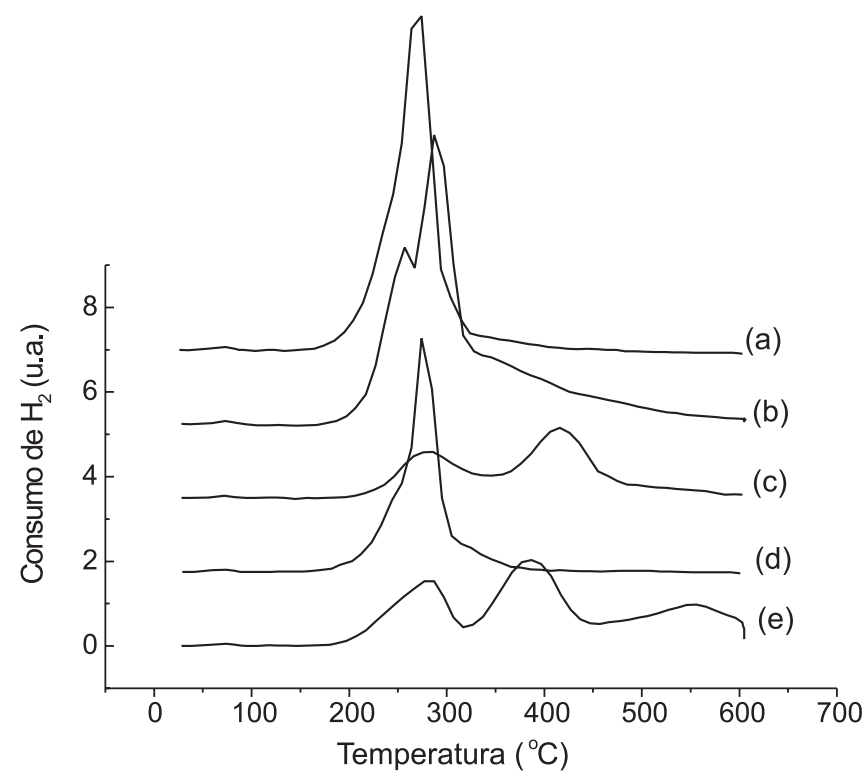

Figura 2. Perfis de RTP dos materiais: a) CuZ50, b) CuZ80, c)CuM30, d) CuM100 e e) CuB25

poderíamos atribuir à redução das espécies $(\mathrm{Cu}-\mathrm{O}-\mathrm{Cu})^{+2}$. Tanto na MCM-22 (CuM30) como na Beta observamos um pico a aproximadamente $400{ }^{\circ} \mathrm{C}$, que poderíamos atribuir à redução de $\mathrm{Cu}^{+} \mathrm{a} \mathrm{Cu}$.

A partir destes dados obtém-se a porcentagem de redução das espécies, apresentadas na Tabela 5. Observa-se uma redução baixa de cobre em todos os catalisadores. O catalisador CuM100 apresentou redução numa ordem de grandeza maior que os demais, provavelmente devido ao baixíssimo teor de cobre, que proporciona uma grande dispersão do íon cobre na amostra.

Tabela 5. Resultados da análise de RTP

\begin{tabular}{|c|c|c|c|}
\hline Catalisadores & $\% \mathrm{Cu}$ & $\begin{array}{c}\text { \% redução } \\
\left(\mathrm{Cu}^{+2} \rightarrow \mathrm{Cu}^{+1}\right)^{\mathrm{a}}\end{array}$ & $\mu \mathrm{mol} \mathrm{H}_{2} / \mathrm{mgCu}^{\mathrm{b}}$ \\
\hline $\mathrm{CuZ50}$ & 3,2 & 2,12 & 0,618 \\
\hline $\mathrm{CuZ80}$ & 2,7 & 3,96 & 0,334 \\
\hline CuM30 & 2,7 & 2,00 & 0,276 \\
\hline CuM100 & 0,21 & 20,00 & 3,149 \\
\hline $\mathrm{CuB} 25$ & 3,3 & 1,76 & 0,316 \\
\hline
\end{tabular}

${ }^{\text {a }}$ Considerando que todo $\mathrm{Cu}^{+2}$ reduziu-se a $\mathrm{Cu}^{+1} ;{ }^{\text {b }}$ consumo total de $\mathrm{H}_{2}$ por cobre total presente no catalisador

Pela facilidade de redução das espécies $\mathrm{Cu}^{+2}$ a $\mathrm{Cu}^{+1}$, provocada pela separação das cargas que ocorre em todos os catalisadores pelo baixo teor de cobre e devido à troca com metal com valência maior que $1, \mathrm{Cu}(\mathrm{OH})^{+}$e $\mathrm{H}^{+}$devem ser os cátions de compensação. Dessa forma é mais provável que esteja ocorrendo uma redução de $\mathrm{Cu}^{+2} \rightarrow \mathrm{Cu}^{+1}$. Assim, são apresentados na Tabela 5 valores de redução de $\mathrm{Cu}^{+2} \mathrm{a} \mathrm{Cu}^{+1}$ (obtidos dos picos a temperaturas menores que $350{ }^{\circ} \mathrm{C}$ ). Não foi considerada a redução total do cobre, que pode estar presente apenas nos catalisadores CuM30 e CUB25 (picos a temperaturas maiores que $350{ }^{\circ} \mathrm{C}$ ). Atribuiu-se a redução de $\mathrm{Cu}^{+2} \mathrm{a} \mathrm{Cu}^{+1}$ aos picos presentes a temperaturas inferiores a $350{ }^{\circ} \mathrm{C}$ e a redução de $\mathrm{Cu}^{+1}$ a $\mathrm{Cu}^{0}$ aos picos presentes a temperaturas superiores a $350^{\circ} \mathrm{C}$, de acordo com atribuições da literatura ${ }^{20-25}$ não foi possível calcular a razão molar $\mathrm{H}_{2} / \mathrm{Cu}$. O perfil de redução dos materiais provavelmente é devido ao processo de redução em etapas $\left(\mathrm{Cu}^{+2} \rightarrow \mathrm{Cu}^{+1} \rightarrow \mathrm{Cu}^{0}\right)$, entretanto, não podemos descartar a possibilidade do cobre estar presente 
em regiões diferentes da zeólita, o que provocaria diversos picos de redução em algumas amostras.

Não foi possível realizar os ensaios de RTP nas mesmas condições empregadas na reação, portanto, estes resultados dão somente uma idéia de quais espécies estariam presentes.

\section{Atividade catalítica}

Os resultados da decomposição do NO mostram que, para os suportes estudados neste trabalho, a melhor conversão é encontrada entre 400 e $450{ }^{\circ} \mathrm{C}$

A conversão em função da temperatura foi estudada para os diferentes suportes zeolíticos e observou-se que as zeólitas MCM-22 e Beta apresentam valores de conversão similares à ZSM-5, como apresentado na Figura 3.

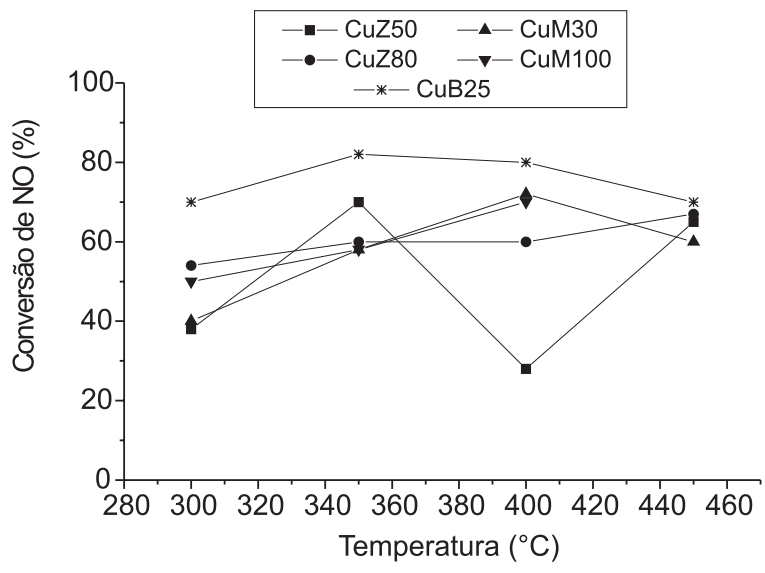

Figura 3. Conversão de NO em função da temperatura no tempo de campanha de 20 min para todos os materiais

Todos os catalisadores apresentaram decréscimo nas atividades catalíticas para a conversão de NO, em função do tempo de campanha bastante acentuado, iniciam entre 40 e $90 \%$ e chegam ao final do tempo de aquisição com $20 \%$. A Figura 4 ilustra este comportamento para a amostra $\mathrm{CuB} 25$ a $350{ }^{\circ} \mathrm{C}$. Todos os testes foram realizados sempre com amostra nova, entretanto para o catalisador CuM30 foi avaliada sua resistência aos testes. Com uma mesma amostra foram realizados os diversos testes catalíticos nas diversas temperaturas. Como resultado, sua atividade foi praticamente a mesma da obtida nos testes empregando amostras novas.

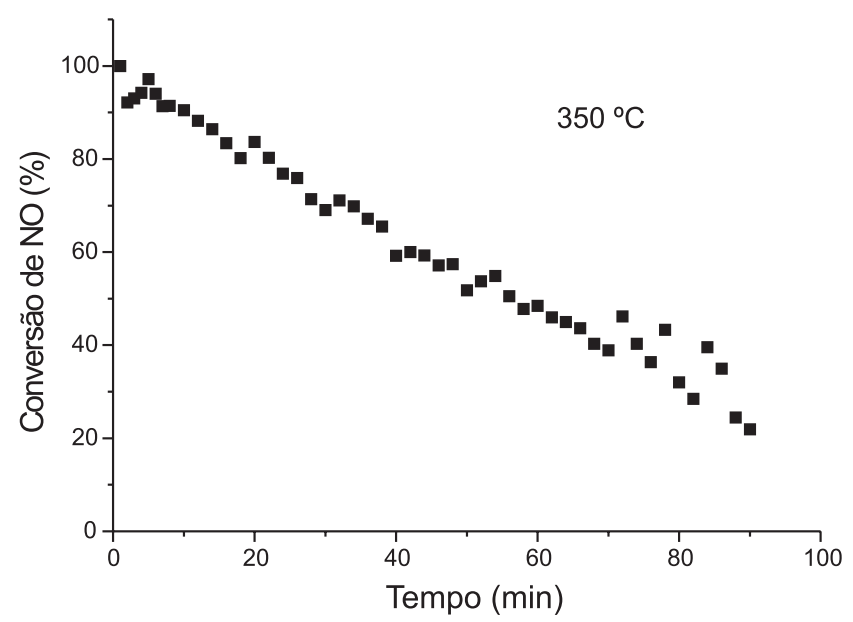

Figura 4. Conversão de NO em função do tempo de campanha para a CuB25 a $350{ }^{\circ} \mathrm{C}$
Embora os catalisadores possuíssem teores similares de cobre, observou-se diferentes atividades, sendo o catalisador $\mathrm{CuB} 25$ o mais ativo. Atribuiu-se tal variação a uma maior acessibilidade da zeólita Beta, que possui poros formados por 12MR, enquanto que as zeólitas MCM-22 e ZSM-5 possuem poros formados por aberturas de 10MR.

$\mathrm{Na}$ decomposição do NO deseja-se que os produtos sejam $\mathrm{N}_{2}$ e $\mathrm{O}_{2}$. Vários mecanismos têm sido propostos para esta reação ${ }^{26-30}$. Uma simplificação dos mecanismos propostos seria que o $\mathrm{NO}$ adsorve na superfície do catalisador e se decompõe em $\mathrm{N}$ adsorvido e $\mathrm{O}$ adsorvido. Os nitrogênios adsorvidos reagem entre si formando $\mathrm{N}_{2}$, o mesmo acontece com o oxigênio.

$\mathrm{NO} \rightarrow \mathrm{NO}_{\text {ads }}$

$\mathrm{NO}_{\text {ads }} \rightarrow \mathrm{N}_{\text {ads }}^{\text {ads }}+\mathrm{O}_{\text {ads }}$

$2 \mathrm{~N}_{\text {ads }}^{\text {ads }} \rightarrow \mathrm{N}_{2 \text { ads }}^{\text {ads }} \rightarrow \mathrm{N}_{2}^{\text {ads }}$

$2 \mathrm{O}_{\text {ads }} \rightarrow \mathrm{O}_{2 \text { ads }} \rightarrow \mathrm{O}_{2}$

Entretanto, outras reações entre as espécies adsorvidas podem ocorrer:

$\mathrm{NO}_{\text {ads }}+\mathrm{N}_{\text {ads }} \rightarrow \mathrm{N}_{2} \mathrm{O}_{\text {ads }} \rightarrow \mathrm{N}_{2} \mathrm{O}$

$\mathrm{NO}_{\text {ads }}+\mathrm{O}_{\text {ads }} \rightarrow \mathrm{NO}_{2 \text { ads }} \rightarrow \mathrm{NO}_{2}$

que dariam como produtos $\mathrm{N}_{2} \mathrm{O}$ e $\mathrm{NO}_{2}$.

A seletividade dos catalisadores à formação de $\mathrm{N}_{2}$ e $\mathrm{O}_{2}$ foi indiretamente avaliada a partir da formação de $\mathrm{N}_{2} \mathrm{O}$ e $\mathrm{NO}_{2}$, devido à impossibilidade de detectar $\mathrm{N}_{2}$ e $\mathrm{O}_{2}$ por infravermelho. A Figura 5
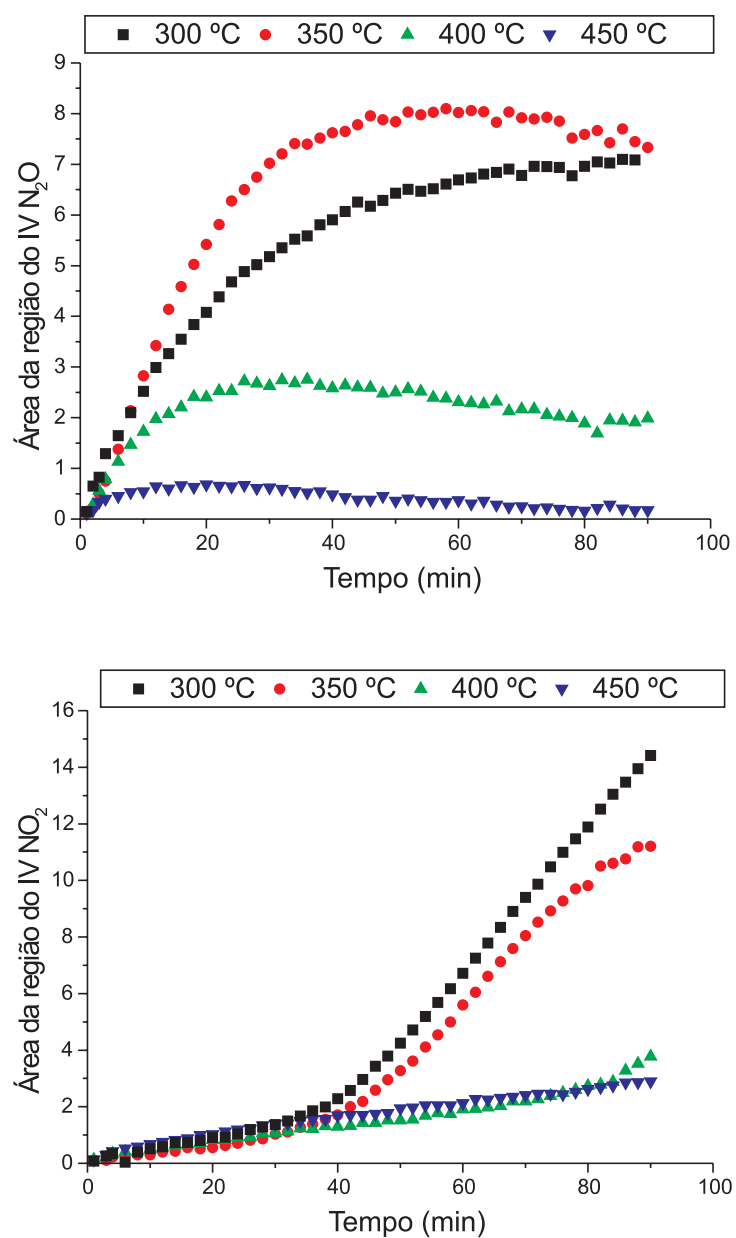

Figura 5. Indicativo de seletividade para a reação de decomposição do NO no catalisador CuM30 
mostra os resultados obtidos para a amostra CuM30. Em temperaturas relativamente baixas $\left(300-350^{\circ} \mathrm{C}\right)$ há formação de grande quantidade de $\mathrm{N}_{2} \mathrm{O}$, fato este que foi evidenciado para todos os catalisadores analisados, sendo que o catalisador $\mathrm{CuZ50}$ apresentou a maior seletividade a tal produto, o que está de acordo com a sua menor atividade para a decomposição de NO a baixas temperaturas.

\section{CONCLUSÕES}

Através da análise de difratometria de raios-X observa-se que não houve formação de agregados de óxidos de cobre na superfície e nas cavidades das zeólitas, pois não foram detectados sinais importantes na região $2 \theta$ igual $36^{\circ}$ e $39^{\circ}$, referentes a $\mathrm{CuO}$ e $\mathrm{Cu}_{2} \mathrm{O}$. Logo, estes catalisadores devem apresentar uma boa dispersão do íon cobre no interior da estrutura zeolítica. As isotermas de adsorção de nitrogênio corroboram tal dado, pois não se notou diferenças de volume de microporos significativas provocadas pela incorporação do cobre. As medidas de atividade mostraram que a melhor temperatura de trabalho estaria na faixa de $400-450{ }^{\circ} \mathrm{C}$, pois há menor formação de $\mathrm{N}_{2} \mathrm{O}$ e de $\mathrm{NO}_{2}$. Nas condições deste estudo, o melhor catalisador pareceu ser o CuB25 na temperatura de $400{ }^{\circ} \mathrm{C}$. Todos os catalisadores preparados apresentaram queda sensível na atividade após alguns minutos de uso. A acessibilidade das estruturas zeolíticas parece ter um papel importante, sendo os materiais mais ativos os que possuem uma estrutura mais acessível.

\section{AGRADECIMENTOS}

Agradecemos à CAPES, à Drª N. R. C. F. Machado pelas análises de redução à temperatura programada e ao Dr. K. Spag pelas análises de adsorção de nitrogênio.

\section{REFERÊNCIAS}

1. Oliveira, A. M.; Dissertação de Mestrado, Universidade Federal do Rio Grande do Sul, Brasil, 2002.
2. Borrego, C.; Miranda, A. I.; Coutinho, M.; Ferreira, J.; Carvalho, A. C.; Environ. Pollut. 2002, 120, 115.

3. Garin, F.; Appl. Catal., A 2001, 222, 183.

4. Marécot, P.; Fakche, A.; Kellali, B.; Mabilon, G.; Prigent, M.; Barbier, J.; Appl. Catal., B 1994, 3, 283.

5. Centi, G.; Perathoner, S.; Appl. Catal., A 1995, 132, 179.

6. Valyon, J.; Hall, W. K.; Catal. Lett. 1993, 19, 109.

7. Shimokabe, M.; Okumura, K.; Ono, H.; Takezawa, N.; React. Kinet. Catal. Lett. 2001, 73, 267.

8. Konduru, M. V.; Chuang, S. S. C.; J. Catal. 1999, 187, 436.

9. Pârvulescu, V. I.; Grange, P.; Delmon, B.; Catal. Today 1998, 46, 233.

10. Argauer, R. J.; Landolt, G. R.; US pat. 37028861972.

11. Rubin, M. K.; Chu, P.; US pat. 49543251990.

12. Leonowicz, M. E.; Lawton, J. A.; Lawton, S. L.; Rubin, M. K.; Science 1994, 264, 1910.

13. Wadlinger, R. L.; Kerr, G. T.; Rosinsk, E. J.; US pat. 33080691967.

14. Iwamoto, M.; Yahiro, H.; Torikai, Y.; Mizuno, N.; Chem. Lett. 1990, 1967.

15. Dallago, R. M.; Tese de Doutorado, Universidade Federal do Rio Grande do Sul, Brasil, 2002.

16. Iwamoto, M.; Yahiro, H.; Tanda, K.; Mizuno, N.; Mine, Y.; Kagawa, S.; J. Phys. Chem. 1991, 95, 3727.

17. Iwamoto, H.; Hamada, H.; Catal. Today 1991, $10,57$.

18. Kharas, K. C. C.; Robota, H. J.; Liu, D. J.; Appl. Catal., B 1993, $2,225$.

19. Moretti, G.; Dossi, C.; Achille, F.; Recchia, S.; Psaro, R.; Appl. Catal., B 1999, 20, 67.

20. Herman, R. G.; Lunsford, J. H.; J. Phys. Chem. 1975, 79, 2388.

21. Iwamoto, M.; Maruyama, K.; Yamazoe, N.; Selyama, T.; J. Phys. Chem. 1977, 81, 622 .

22. Mendes, F. M. T.; Dissertação de Mestrado, Universidade Federal do Rio de Janeiro, Brasil, 1995.

23. Sárkány, J. D. J. L.; Sachtler, W. M. H.; Catal. Lett. 1992, 16, 241.

24. Tanabe, S.; Matsumoto, H.; Appl. Catal. 1989, 47, L9.

25. Hurst, N. W.; Gentry, S. J.; Jans, A.; Catal. Rev. - Sci. Eng. 1982, 24, 233.

26. Shimokawabe, M.; Okumura, K.; Ono, H.; Takezawa, N.; React. Kinet. Catal. Lett. 2001, 73, 267.

27. Li, Y.; Armor, J. N.; Appl. Catal. 1991, 76, L1.

28. Garin, F.; Appl. Catal., A 2001, 222, 183.

29. Giamello, E.; Murphy, D.; Magnacca, G.; Morterra, C.; Shioya, V.; Nomura, T.; Anpo, M.; J. Catal. 1992, 136, 510.

30. Aylor, A. W.; Larsen, S. C.; Reimer, J. A.; Bell, A. T.; J. Catal. 1995, 157, 592. 